\title{
LOGICAL THEORY REVISION THROUGH DATA UNDERDETERMINATION: AN ANTI-EXCEPTIONALIST EXERCISE
}

\author{
SANDERSON MOLICK \\ Federal University of Rio Grande do Norte (UFRN), BRAzIL \\ Ruhr-University of Bochum (RUB), GERMANY \\ smolicks@gmail.com
}

\begin{abstract}
The anti-exceptionalist debate brought into play the problem of what are the relevant data for logical theories and how such data affects the validities accepted by a logical theory. In the present paper, I depart from Laudan's reticulated model of science to analyze one aspect of this problem, namely of the role of logical data within the process of revision of logical theories. For this, I argue that the ubiquitous nature of logical data is responsible for the proliferation of several distinct methodologies for logical theories. The resulting picture is coherent with the Laudanean view that agreement and disagreement between scientific theories take place at different levels. From this perspective, one is able to articulate other kinds of divergence that considers not only the inferential aspects of a given logical theory, but also the epistemic aims and the methodological choices that drive its development.
\end{abstract}

Keywords: Logical revision • anti-exceptionalism about logic $\bullet$ paraconsistency $\bullet$ logical pluralism

\section{Introduction}

Logical anti-exceptionalism is the view that logical and scientific theories are alike (Hjortland 2017). From a philosophy of science perspective, this means that the descriptive models of the processes of development and formation of disagreements and agreements between different scientific theories can be used to describe analogous processes among disputant logical theories. Therefore, given that different models are available in the philosophical literature, ${ }^{1}$ an important problem is to know what sort of model best represents agreement and disagreement among logical theories. In the following, I explore this problem based on Laudan (1984) reticulated model of science.

Laudan's reticulated model allows one to explore distinct commitments carried by advocates of a scientific theory through the integration of methodological and factual elements of the theory. The processes of agreement and disagreement formation are described through the dynamics of discovery of new data, production of suitable 
heuristic mechanisms, and pursuit of selected epistemic values. The resulting picture combines theoretical elements treated by other philosophers of science such as Lakatos and Popper.

In the present paper, I depart from a representational conception of logical theories, which considers not only the inferential apparatus, but also the representational aims in view of their methodology and their epistemic goals. This representational view draws from other approaches initiated by, for instance, Thagard (1982), Aberdein \& Read (2009), and Hjortland (2019). However, whilst Aberdein \& Read (2009) focus on the inferential aspects of a logical theory, such as the revision of syntax and semantics of base logical systems, the following approach focuses on the role of the logical data in triggering different types of agreement and disagreement.

The upshot of the paper is a descriptive approach to logical theories that fits Laudan's reticulated model and shows how the very mode of representation of the logical data is responsible for giving rise to specific forms of methodological disagreements between logical theories. The underdetermined character of logical data is shown to play a significant role in the proliferation of distinct methodologies. Accordingly, data underdetermination shall not be understood as a global phenomenon of empirically equivalent evidence for rival theories, but rather as a local phenomenon of existing equally plausible interpretations for a given set of data.

The paper is structured as follows: in Sections 2 and 3, I introduce two different ways of looking at logical theories, namely as inferential devices and as representational structures. I discuss how the latter view relates to logical anti-exceptionalism and the advantages of this notion of logical theory. The paper proceeds to Section 4 , where the proposed conception of logical theory is analyzed according to Laudan (1984) reticulated model of justification for scientific theories. In Section 5 the problem of the underdetermination of the logical data is explored in relation to the reticulated view on justification. The underdetermined feature of logical data is illustrated in Section 6 through the case of paraconsistent theories and the debate over adequate interpretations of the concept of contradictions. Different types of disagreements are shown to play a significant role in the process of revision of distinct paraconsistent theories. The paper continues to Section 7 by exploring different forms of pluralism arising from the reticulated view on the formation of agreement and disagreement.

\section{Logical theories as inferential devices}

A logical theory is an inferential structure capable to determine which consequences are valid in view of an underlying notion of logical consequence. In rough terms, a logical theory is defined by the set of valid inferences relative to a logical system and a set of basic sentences. Given a base logical system $\mathrm{L}=\langle\mathscr{S}, \vdash\rangle$, where $\mathscr{S}$ is a set 
of formulas and $\vdash \subseteq \wp(\mathscr{S}) \times \mathscr{S}$ is a consequence relation, let the closure of a set of formulas $\Gamma \subseteq \mathscr{S}$ be $\Gamma^{\vdash}=\{\alpha: \Gamma \vdash \alpha\}$. A logical theory $\mathscr{T}$ is defined as the following resulting structure:

$$
\mathscr{T}=\left\langle\Gamma, \Gamma^{\vdash}\right\rangle
$$

Call the representation above the purely inferential view on logical theories. From the fact that a logical theory is grounded on a logical system, the theory is said to be an expression of the conception of validity intrinsic to the logical system. Hence every logical theory can be seen as a theory of validity. They determine which inferences are valid in view of a suitable consequence relation. Two logical theories disagree whenever one accepts as valid an inference, say $p$, denied by the other. In such a case, a logical disagreement is said to happen. As Hjortland (2019, p.252) put this, "an account of logical theories should therefore reflect that there are rival theories that disagree on the validity of arguments".

In contemporary logic, logical disagreements are a common phenomenon due to the proliferation of logical systems. But if so many different theories of validity are available, a straightforward problem is to know when and how to choose one among the many. The anti-exceptionalist ${ }^{2}$ offers an apparently simple solution: assume logical theories are akin to scientific theories, then a decision procedure can be employed just like it happens with the sciences. Start by selecting a set of relevant data and measure the vices and virtues of each logical theory, the best scoring ones ought to be chosen. In order to perform this weighing procedure, the relevant criteria shall be those employed by the hard sciences: adequacy to the data, fruitfulness, simplicity, consistency, elegance, and so on.

The anti-exceptionalist move seems harmless, but it comes with a price. To say that logical and scientific theories are alike is to say they are both fallible endeavors, which are subject to a process of revision through the employment of formal tools in the description of a wide range of phenomena. And if the content of logical theories is taken to bear non-aprioristic features, it means that logical theories are also representational structures. They model some form of data upon which they are also supposed to account and explain. As a result, we move from a conception of logic as a theory of deduction to a conception of logic-as-modelling, where now the inferential apparatus ought to be tested against a specific kind of data. ${ }^{3}$ Moreover, for a phenomenon to be described by a logical theory, the inferential apparatus does not seem to suffice in order to decide which theory stands as the most adequate. Representational features related to the relevant domain of application, to how to best represent it, and to how the data ought to be accounted for, shall play a significant role in this.

Consider the logical dispute between classical and intuitionistic theories. It is well known that the core of this debate is centered around the unrestricted validity of the 
law of excluded middle in classical theories. ${ }^{4}$ If we compare them from an inferential perspective by analyzing virtues such as strength and simplicity, it is clear that classical theories have a head start on intuitionistic theories. However, once we select a specific domain of application, namely of constructive sets and proofs, the epistemic virtues selected by the intuitionist such as constructiveness and effectiveness shall start to balance the scale for intuitionistic theories. Soon, the disputants shall need to make explicit the reasons why one's epistemic aims are better than others, and their philosophical commitments will surface the discussion.

When comparing logical theories on purely inferential grounds, representational aspects such as the epistemic aims of the theory, the methods for exploring the selected data and the intended domain of application are often neglected. Accordingly, an important methodological aspect adopted throughout the present paper is to propose and explore a broader conception of logical theory that is able to articulate inferential and representational elements within the process of revision of a logical theory. To explore the relation between inferential and representational elements is to make explicit underlying methodological and philosophical commitments.

To evaluate logical theories in view of their representational capacities allows their treatment as dynamical structures sensitive to revisional processes. The epistemic goals and the philosophical commitments of the theory are both put into play, and not treated as extra-logical elements orthogonal to the deductive apparatus. Considerations over the heuristic mechanisms, methods and constraints that guide the development of the theory are assumed to play an important role. From this perspective, logical theories are understood as structures within research programs to mine distinct domains of logical data. Naturally, an important problem is to know what should count as logical data. For this I shall follow the anti-exceptionalist move of considering a wide range of phenomena, from mathematical constructions to natural language phenomena, to be coherent with the common practice in the logic literature.

\section{Logical theories as representational structures}

Now, in order to consider representational features of a logical theory, the purely inferential conception needs to be further qualified. These representational features are often described as mechanisms to achieve the deductive goal of the system. Thus, many elements may be considered, like the intended semantics of the base system, its metatheory, or even types of deductive mechanisms. However, in order to focus only at the modelling features of a logical theory, I shall focus on two main aspects: the relevant data to be accounted for, and the methodological grounds for exploring the relevant data. For this, I shall call the representational apparatus of a logical 
theory the following structure:

$$
\mathrm{R}_{\mathscr{T}}=\langle\text { METHODOLOGY, INFERENTIAL GOAL }\rangle \text {, }
$$

where $\mathscr{T}$ is a logical theory. The methodology of a logical theory shall be called any set of formal and philosophical constraints developed within a research program to achieve the inferential goal of the theory. ${ }^{5}$ The role of the inferential goal is to prescribe what the inferential practice is intended to achieve and what criterion plays a role in delimiting valid from invalid inferences. The most common example is the preservation of truth, but many logical theories require additional constraints to the validity of arguments, such as relevance, preservation of warrant, non-triviality in face of inconsistencies, and others alike (Aberdein \& Read 2009).

In the process of development of a logical theory the inferential goal is often established as a reaction to some anomalous data to the reference theory. The deviant logical theory ought to promote a new inferential goal capable to accommodate (or reject) the anomalous data. In this process the inferential goal determines the epistemic goals of the deviant theory motivated by the selection of a relevant set of data. Thereafter the methodology shall start to develop a set of constraints for the adequate exploration of the data. For example, the methodology may establish that the relevant data shall be treated as a semantic phenomenon of natural language, and for this a non-distributive conjunction must be taken as a minimal requirement for its adequate description. Soon adequate syntactical and semantical constraints ought to be defined in order to produce logical systems in accordance with all the restrictions chosen by the methodology. As a result, the representational apparatus of the deviant theory becomes responsible for guiding the development of suitable inferential tools.

Four elements shall be assumed to play a key role in the dynamical character of logical theory revision: 1) the logical systems, 2) their semantics, 3) the methodology, and 4) the inferential goal. Based on this, a descriptive conception of a logical theory may be introduced as:

$$
\mathscr{T}_{d}=\langle\text { LOGICAL SYSTEM, SEMANTICS, METHODOLOGY, INFERENTIAL GOAL }\rangle .
$$

From the descriptive point of view, it is easy to see that revising the logic and revising the theory should not be conflated. Whereas the revision of the logic occurs in the base system and its underlying notion of validity, the revision of the theory may occur at other elements. This paper also distinguishes between revision and deviation: the former shall be understood as internal changes to logical theories, while the latter as the inferential departure from the reference theory (here assumed to be classical logic). Deviation, then, may be expressed as a disagreement over the inferential goal. However, logical theories may diverge by considerations on any of the abovementioned four elements. 
In Aberdein \& Read (2009), the authors explore many sources of divergence resulting from elements (1) and (2) above. In the present paper, I shall explore types of divergence related to elements (3) and (4) only. For this, the ubiquitous nature of the logical data shall play a key role in the process of revision of a logical theory. In this setting, logical revision is understood as the many adaptations a logical theory may suffer in the effort to adequately describe a relevant set of data. The process of logical theory revision is understood as the struggle to produce adequate inferential and representational tools to model a relevant phenomenon. ${ }^{6}$

\section{A bird's eye view: insights from Laudan}

In the philosophy of science, an influential model for description of the process of formation of agreement and disagreement between scientific theories was proposed by Laudan (1984) in response to some irrationalist threats perpetrated by Kuhn and to the hierarchical view of the process of justification of scientific theories. Laudan's model, called reticulated model of justification, is based on the idea that scientific theories may disagree at three distinct levels: factual, methodological, and axiological.

At the factual level there are all types of claims about reality, "including claims about theoretical or unobservable entities" (Laudan 1984, p.23). Debates of this sort are called factual disagreements, and agreements of this sort are called factual agreements. Factual disagreements involve not only debates about the truth or falsity of certain claims, but also about the relevant data to be explained and the facts to be accounted for. According to the reticulated model, factual disagreements can be settled at the previous level, the methodological level. In its turn, the methodological level is determined by considerations over what are the best procedures to explore and explain the data at hand, where these methodological choices are guided and influenced by considerations from the preceding level, the axiological level. In Laudan's terms, the axiological level may be described as the set of cognitive/epistemic goals established by the theory, e.g. simplicity, adequacy to the data, consistency, and so on. Given an agreement over a set of epistemic goals, the relation between the methods and the axiology may be described in terms of conditionals of the kind: "If one wants to accomplish such and such cognitive goal, then she ought to act according to such and such methods".

In Laudan's reticulated model, the process of agreement and disagreement occurs back-and-forth among all different levels. New intuitions about the data may lead to revisions at the axiological level. Thereafter new methodological constraints might be considered in order to perform the new goals determined by the axiological level. Similarly, new intuitions about the cognitive goals might lead to revisions at the factual level through novel considerations about the relevant data. As acknowledged 
by Laudan (1984, p.35): “(...) cognitive [epistemic] aims typically underdetermine methodological rules in precisely the same way that methodological rules characteristically underdetermine factual choices." This intertwined feature across all levels leads to several types of adjustments relative to each level. Thus, scientific theory revision is the result of looking for an equilibrium state among the three different levels motivated by a constants process of agreement and disagreement formation at each level.

Back to logical theories, the reticulated view on justification provides an assessment of the relation among the distinct elements of the descriptive conception of logical theories. Among the factual disagreements, there are disagreements about the logical data at hand. As discussed before, deviance from the reference theory is motivated by an inferential disagreement due to allegedly anomalous data. Such inferential disagreement is taken to reflect an incompatibility among the epistemic goals of the disputant theories. Therefore, the inferential disagreement between rival theories is understood as resulting from both an axiological dispute over adequate epistemic goals, and from a factual dispute over the relevant set of data. From this, a new inferential goal to handle anomalous data is required from the deviant theory. Naturally, after the establishment of a new inferential goal, a set of methodological constraints will be adapted for the logical theory at stake.

The intertwined character across the elements of a logical theory is present in the processes of revision and deviation. Disputes over the adequate data may lead to disputes over the adequate inferential goal. Conversely, considerations over the inferential goal may lead to methodological disputes over the adequate manner to represent the selected data. In the same way, new intuitions over the data may lead to novel refinements of the inferential goal. As analogous to the reticulated model, the back-and-forth process of revision of a logical theory is fed by the pursuit of an adequate representation of a relevant phenomenon with the most suitable inferential tools.

The resulting picture agrees with the reticulated model in the following way:

\begin{tabular}{l|l} 
Levels of Disagreement & Logical theory \\
\hline C. Factual & Data \\
B. Methodological & Meth. constraints \\
A. Axiological & Inferential goal
\end{tabular}

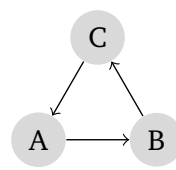

As exhibited in the picture above, the reticulated view accommodates different types of disagreement. Even if a deviant logical theory diverges from the reference theory due to the validity of a certain inference, say the idempotence of conjunction, partisans of the deviant theory may still as well disagree over factual and method- 
ological issues. Whereas one group might prefer to understand the relevant logical data as representing epistemic situations and to produce logical systems with a truthfunctional semantics, another group might prefer exploring an ontological conception of the logical data, guided by a methodology that privileges non-truth-functional adequate logical systems.

As an example, think of the case of paraconsistent theories. Once the inferential goal of rejecting triviality in face of contradiction is established, the methodology ought to determine specific constraints in order to produce adequate logical systems, such as the presence of a non-explosive negation, or a non-adjunctive conjunction, and so on. The different ways to achieve the epistemic goal proliferate distinct methodologies and research traditions around the shared goal. As mentioned, however, even if paraconsistentists do agree over the inferential goal, they might still disagree over factual and methodological matters. In the following, I discuss how these distinct types of disagreements are triggered by the very nature of the logical data.

\section{The role of the logical data}

In science, the revision of a theory may occur by the discovery and exploration of anomalous data that do not fit within the reference theory, as well as by extraempirical reasons discovered in the process of solving problems that are internal to the theory in order to enhance virtues such as scope, coherence, explanatory power, or applicability. ${ }^{7}$ In a similar manner, revisions and deviations of classical logic were suggested as the outcome of different kinds of disagreements resulting from the applicability of the inferential apparatus to distinct domains, as well as from the will to extend the scope of application of classical principles.

In the logic literature, different cases for deviance and revision were presented. Paraconsistent logicians called for the revision of the principle of explosion due to the discovery of semantic and set-theoretic paradoxes; quantum logicians called for the revision of the law of distributivity in reaction to the empirical discoveries of quantum mechanics; intuitionistic logicians called for the revision of the law of excluded middle on account of concerns with the constructive character of mathematical reasoning; plural logicians called for adequate expressive power to formalize plural quantification as in natural language, and others alike. The defenders of each of those positions shared at least one common purpose, i.e. to revise some underlying feature of classical logic by either disputing the validity of a certain inference or by extending its deductive strength.

Whereas some of these arguments attacked the inadequacy of classical logic to describe some empirical phenomena, others appealed to some inferences that seemed 
not in accordance with our day-to-day practices, or even against the metaphysical picture implied by some classical principles. Therefore, our revisionary practices seem to follow the rationalist tenets according to which the reference theory ought to be subject to critical examination and put to the test of a wide range of phenomena. This seems to be in line with Hjortland's intuition about what types of logical data are acceptable for anti-exceptionalist purposes:

The evidence for a logical theory can come from a number of different sources: from intuitions about validity or alethic modality, from mathematical theories and practice, from psychology of reasoning, from epistemic norms of rationality, and so on (Hjortland 2017, p.644).

From Hjortland's description one may note that logical data may be assumed to spring from observational and non-observational sources. This ubiquitous character of logical data gives logical inferences a high degree of verifiability for it makes them susceptible to different interpretations of what sort of data they bear on. As a result, the underdetermination of the logical data leads to equivocal interpretations of the logical inferences in dispute. Whereas an attacker of a logical principle may provide epistemic situations as evidence against its validity, others may present as diverse phenomena as mathematical constructions, physical events, semantical constructions, informational processes, etc. ${ }^{8}$ Different views of classical logic seem to result from this, namely of:

- Classical logic as a set of descriptions of linguistic phenomena. This form of revision is motivated by interpreting classical validities as reflecting linguistic structures. Therefore, linguistic data are provided as anomalies to the principle in dispute.

- Classical logic as a set of rationality principles. In this kind of revision, the opponent does not agree with how classical principles model a certain inferential situation. Logical principles are taken to be rules of procedure that guide agents through different inferential situations. Accordingly, epistemic situations are provided as anomalous data to the principle in dispute.

- Classical logic as a set of metaphysical truths. In this kind of revision, the opponent attacks some metaphysical presupposition implied by the principle under revision. Logical principles are hence taken to be metaphysical principles and therefore to characterize some fundamental structures of reality.

All positions above result from distinct perspectives over the content of classical principles and what type of domain they ought to describe. If deviations begin with the exploration of anomalous data, in order to develop coherent guiding principles, the methodology of the new theory has to answer the following two questions about 
the nature of the logical data at hand: 1) What domain of our experience does the data represent?; and 2) What principles of the reference logical theory does the logical data conflicts with? The answer to these questions is the key for establishing how the deviant logical theory interprets the content of the reference theory. In the following, I explore the case of paraconsistent logics in order to analyze how these different views of classical principles lead to different methodologies. The underdetermined character of logical data is exhibited through how methodological disputes emerge around paraconsistent theories.

\section{Paraconsistency and kinds of disagreement}

The birth of paraconsistent logics is marked by the discovery of new logical data stemming from mathematical and semantical theories. The discovery of paradoxes, semantical and set-theoretical, provoked the concern with the development of alternative ways to handle inconsistent theories without falling into triviality. ${ }^{9}$ As a result, this sort of phenomena led to a general dissatisfaction with the principle of explosion (PE) and the methodological attitude induced by the classical logician in face of contradictions. $^{10}$

The cornerstone of the methodology of paraconsistent logics was developed around the requirement of preventing the explosive behavior of classical negation. The variety of techniques developed to meet such a requirement blossomed into the high number of paraconsistent logics available in the literature, labelled into distinct categories and methodological traditions. However, from the fact that paraconsistentists share the same inferential goal, namely avoiding triviality in face of contradictions, one cannot conclude that they all share the same methodological and factual beliefs. An important point of contention among paraconsistentists occurs at the factual level of paraconsistent theories, with side-effects on their methodology. These disputes are relative to the nature of contradictions, to what kind of items contradictions are, as well as to what sort of explanatory power they provide to paraconsistent theories. Whereas some paraconsistentists (like Mares (2004)) regard contradictions as a mere semantic or linguistic phenomenon with low explanatory power to our theories of reality, others (like Priest (2006)) take them to reflect ontological features of the world.

As discussed in Section 4, according to the reticulated model, new insights over the epistemic goals might be motivated by novel insights at the factual level. This means that distinct forms of looking at the data may lead to new intuitions about how to understand the epistemic aims of the theory. In the case of logical theories, the same process of revision happens by discovering new ways of interpreting the inferential goal of the theory in light of novel insights about the data under consideration. 
Accordingly, new insights over how to adequately interpret the inferential goal of the theory may lead to different interpretations of the logical vocabulary. As examples, in Wansing \& Odintsov (2016) the authors defend information as the relevant data for paraconsistent theories so that paraconsistency can be motivated independently of epistemological or metaphysical commitments. According to this view, negation is understood as an operation of cancellation, and the failure of PE is explained by assuming that from the information of $A$ and $\neg A$, one cannot infer any information about $B$. In an analogous manner, Carnielli \& Rodrigues $(2012 ; 2019)$ argue for the view that contradictions have only an epistemic character, and that they cannot be taken as space-time phenomena, nor as mathematical objects. In their Evidence-LFIs systems, evidence is assumed to be the relevant data for paraconsistent theories and the failure of $\mathrm{PE}$ is motivated by the idea that non-explosive contradictions denote only conflicting non-conclusive evidence.

The selection of the relevant data is then accompanied by an interpretation of the principle in dispute with the reference theory. In the case of the rivalry between paraconsistent and classical theories, four different views on inconsistencies may be distinguished: epistemic (Carnielli \& Rodrigues 2019), semantic (Priest (2014) and Mares (2004)), ontological (Priest 2006), and informational (Wansing \& Odintsov 2016). ${ }^{11}$ The interpretation of inconsistencies for epistemic purposes is motivated by comprehending classical logic as a set of rationality principles that are useful to organize collections of knowledge and evidence in the process of scientific investigation. In this case PE is understood as a prohibition to accept contradictory evidence, and negation is read in terms of incompatibility. Authors who favor the epistemic view usually weigh the explanatory power of paraconsistent theories through the description of epistemic faculties such as knowledge, evidence, justification, belief, and others. $^{12}$

The informational perspective on contradictions is also similar to the epistemic one, but in the sense of Wansing \& Odintsov (2016) it has the purpose of ruling out considerations about epistemic agents. As explained by the authors:

With the conception of logic as the theory of valid inferences and the conception of logics as consequence relations, logic, by definition, is committed to the existence of languages but not necessarily to the existance of language users.(...) Moreover, the notion of valid inference does not refer to the knowledge or belief states of any epistemic or doxastic subjects. (Wansing \& Odintsov 2016, p.180)

In a different manner, the view on contradictions as semantic items is motivated by an interpretation of classical logic as a set of descriptions for linguistic phenomena. For this view inconsistencies arise due to the vagueness of certain concepts. Classical logic is seen as a faulty description of our linguistic practices. Examples of 
a semantic view on contradictions are provided by Mares (2004) and Priest (2014) through overlapping linguistic concepts. Lastly, the ontological view is characterized by taking inconsistencies to represent aspects of reality. Classical logic is understood as a set of metaphysical truths, and the paraconsistentist fights $\mathrm{PE}$ as a consequence of challenging the law of non-contradiction as a metaphysical principle. ${ }^{13}$

\begin{tabular}{lll} 
Principle & Data & Interpretation \\
\hline \hline \multirow{3}{*}{$A, \neg A \vdash B$} & Informational & "From the information of $A$ and $\neg A$ one can infer information about $B "$ \\
& Epistemic & "One cannot hold contradictory evidence without falling into triviality" \\
& Ontological & "Reality cannot comprise contradictory items" \\
& Semantic & "Language cannot hold contradictory concepts"
\end{tabular}

Table 1: Different views on contradictions

The resulting views are summarized in Table $1 .{ }^{14}$ It shows how the inferential disagreement over the validity of the principle of explosion may be motivated by different specifications of the logical data. The content of PE is made precise in different ways by exploring distinct kinds of data. As a result, distinct interpretations over the inferential goal of rejecting PE bring about different methodologies for the development of suitable logical systems. For each kind of data at stake, different tools shall be developed in order to avoid the unrestricted validity of PE. ${ }^{15}$ Whereas the informational reading of PE may avoid its validity through constraints of relevance, the semantic and epistemic readings may avoid its validity through exploring weaker notions of incompatibility.

The different ways of coping with the data lead paraconsistent theories to incur in a methodological underdetermination of their theories by the available evidence. Underdetermination is understood in this context as a local phenomenon due to the existence of equally plausible interpretations of the selected evidence. ${ }^{16}$ As we shall discuss, this type of underdetermination leads to axiological disagreements about whether there is one correct methodology for the development of paraconsistent theories. To state the existence of one correct methodology is to defend the existence of one correct way of interpreting the relevant data, as well as one better set of constraints for the development of suitable logical systems.

To sum up, the representational view on logical theories is coherent with the reticulated dynamics according to which inferential agreements might be attached to matters of how and what is being represented. The underdetermined character of the logical data leads to important disagreements for the methodology of logical theories. A similar sort of data underdetermination also arise in the debate about vagueness. The offered solutions to the body of evidence leads to equally plausible and dual interpretations, such as the subvaluationist and supervaluationist solutions. Whereas the former interprets vague statements as truth-value gluts, supervaluation- 
ists takes vague statements to represent truth-value gaps. Therefore, the different ways of interpreting the data give rise to distinct methodological choices. Hence, distinct traditions emerge as the result of methodological disputes over the adequate manner of exploring the relevant data.

In the following section, I discuss how the different levels of agreement and disagreement leave room for distinct forms of pluralism. These types of pluralism, naturally, are not related to inferential debates over consequence relations, but rather to normative views over adequate constraints for logical theories. They are straightforward consequences of the fact that the reticulated view describes not only factual debates about the truth or falsity of certain claims, but also debates over representational issues.

\section{Beyond inferential pluralism}

As discussed in the previous sections, the reticulated model expresses the idea that different types of debate take place between the defenders of distinct scientific theories. In the case of logic, the relevant debate is not only relative to the truth or falsity of factual claims, but also to debates about the methodological tools, the epistemic goals of the theory, as well as the relevant sets of data.

According to the reticulated view, the existence of agreements between different logical theories about inferential or factual claims does not imply the peaceful coexistence of these theories. Therefore, even if different logical systems agree at the inferential level on the validity of the Tarskian metaproperties of their consequence relations, as suggested by Beall \& Restall (2006), they can still dispute at the methodological or the factual level. If the logical pluralism debate is characterized by a disagreement relative to our inferential views on logical consequence, then two other types of normative debates seem to result in accordance with the reticulated view, namely: the debate over the adequate methodological constraints relative to a suitable inferential view, and the debate over the relevant set of data for logical theories. The former may be summarized by the following positions:

- Methodological pluralism: Different methodologies lead to different perspectives and explanations over the same set of data. The proliferation of methodologies is a positive phenomenon, for it represents ways of exploring and explaining the same set of relevant explanans chosen by the inferential goal. Examples of methodological pluralists are paraconsistentists like da Costa et al. (2007) or Batens (2017), for which there is no best set of constraints to produce paraconsistent systems. The pluralist attitude of this position is also marked by the inclination to accept the use of classical concepts at the metalanguage level, as well as by the will to explore distinct domains of data. 
- Methodological monism: There is only one correct methodology for exploring a given set of data. This one best methodology is usually tied to a fixed interpretation of the relevant logical data. In Wansing \& Odintsov (2016) the authors defend a monist perspective over the methodology of paraconsistent logic, according to which paraconsistent systems ought to be produced by accepting the notion of information as the relevant data along with the rejection of classical logic as the reference logic for paraconsistent theories.

Another type of pluralism coherent with the reticulated model concerns factual disagreements over the acceptable sources of data for logical theories. Here the relevant content for logical principles plays a key role in defining the adequate domain of application of the theory and the role of the logical system as a foundation for it. The debate may be described by the following positions:

- Data source pluralism: Data for logical theories may come from different sources. Logical theories then ought to be foundational to diverse domains of knowledge. This view is shared for instance by Routley (1980). It is important to highlight that even though Routley is in favor of a form of data source pluralism, he is an inferential monist that also supports a forma of methodological monism with respect to paraconsistent theories. Routley's position results from a defense of his ultralogic as the true logic for foundations across all domains of knowledge.

- Data source monism: There is only a single class of relevant data for logical theories. The common examples are the data coming from mathematical theories, from our theories of truth, or from our linguistic practices. For data monists logical theories ought to be foundational to a specific research activity, be it mathematics, linguistics, etc.

\section{Conclusion}

In the present paper, I have explored how the representational anti-exceptionalist account of logical theories fits Laudan's reticulated view of scientific agreement and disagreement. The underdetermined nature of logical evidence was shown to play a significant role in the process of revision and deviation of logical theories. In the process of revision, logical evidence is responsible for allowing the proliferation of distinct methodologies that drive the development of logical theories. On the other hand, in the process of deviation, logical evidence is responsible for allowing different interpretations of the principles in dispute. Logical theory revision is understood through the dynamics of the adequate representation of some kind of data in accordance with suitable methodological tools. The resulting picture is coherent with a 
view in which each logical tradition is developed in pursuit of some selected epistemic goals.

Some topics remain open for further exploration, such as how to characterize deep disagreements in view of the reticulated model, what other types of non-classical theories could be described through the underdetermination of the data, as well as how to characterize the type of logical pluralism in accordance with Laudan's own philosophical views.

\section{References}

Aberdein, A.; Read, S. 2009. The Philosophy of Alternative Logics. In: Leila Haaparanta (ed.) The Development of Modern Logic, pp.613-723. Oxford University Press.

Batens, D. 1999. Inconsistency-adaptive logics. In: Ewa Orłowska (ed.) Essays dedicated to the memory of Helena Rasiowa, pp.445-72. Springer.

Batens, D. 2017. Pluralism in scientific problem solving: why inconsistency is no big deal. Humana.Mente 32(1): 149-77.

Beall, J.C.; Restall, G. 2006. Logical Pluralism. Oxford University Press.

Blake-Turner, C.; Russell, G. 2018. Logical pluralism without the normativity. Synthese DOI: 10.1007/s11229-018-01939-3.

Carnielli, W.; Rodrigues, A. 2012. What contradictions say (and what they say not). CLE ePrints 12(2).

Carnielli, W.; Rodrigues, A. 2019. An epistemic approach to paraconsistency: a logic of evidence and truth. Synthese. 196(9): 3789-3813.

Da Costa, N.; Béziau, J.Y-.; Bueno, O. 1995. Aspects of paraconsistent logic. Logic Journal of the IGPL 3(4): 597-614.

Da Costa, N.; Krause, D.; Bueno, O. 2007. Paraconsistent logics and paraconsistency. Philosophy of Logic 5: 655-781.

Haack, S. 1974. Deviant Logic: Some philosophical issues. Cambridge University Press.

Hjortland, O. T. 2017. Anti-exceptionalism about logic. Philosophical Studies 174(3): 631-58.

Hjortland, O. T. 2019. What counts as evidence for a logical theory? The Australasian Journal of Logic 16(7): 250-82.

Laudan, L. 1978. Progress and its problems: Towards a theory of scientific growth. University of California Press.

Laudan, L. 1984. Science and values: The aims of science and their role in scientific debate. University of California Press.

Lakatos, I. 1978. The methodology of scientific research programmes. In: Philosophical papers, vol.1. Cambridge: Cambridge University Press.

Mares, E. D. 2004. Semantic dialetheism. In: G. Priest; JC Beall; B. Armour-Garb (eds.) The Law of Non-Contradiction: New Philosophical Essays, pp.264-75. Oxford University Press.

Priest, G. 2002. Paraconsistent logic. In: Dov Gabbay \& Franz Guenthner (eds.) Handbook of Philosophical Logic, vol.6, pp.287-393. Springer.

Priest, G. 2006. In Contradiction. Oxford University Press. 
Priest, G. 2014. Contradictory concepts. In: Erik Weber; Dietlinde Wouters; Joke Meheus (eds.) Logic, Reasoning and Rationality, pp.197-215. Springer.

Rosenberg, A. 2011. Philosophy of Science: A contemporary introduction. Routledge.

Routley, R. 1980. The choice of logical foundations: non-classical choices and the ultralogical choice. Studia Logica 39(1): 77-98.

Stanford, K. 2017. Underdetermination of scientific theory. In: E. Zalta (ed.) The Stanford Encyclopedia of Philosophy(Winter 2017 Edition). https://plato.stanford.edu/archives/ win2017/entries/scientific-underdetermination/

Tahko, T. E. 2009. The law of non-contradiction as a metaphysical principle. Australasian Journal of Logic 7: 32-47.

Thagard, P. 1982. From the descriptive to normative in psychology and logic. Philosophy of Science 49(1): 24-42.

Wansing, H.; Odintsov, S. 2016. On the methodology of paraconsistent logics. In: Holger Andreas; Peter Verdée (eds.) Logical Studies of Paraconsistent Reasoning in Science and Mathematics, pp.175-204. Springer.

\section{Notes}

${ }^{1}$ See e.g., Rosenberg (2011)

${ }^{2}$ See Hjortland (2017).

${ }^{3}$ Note one might still prefer only a theory of deduction in order to rule out any consideration over the representational features of a logical theory. However, the present paper shall focus on the representational aspects of logical theories. See Hjortland (2019) for the difference between logic as a theory of deduction and logic-as-models.

${ }^{4}$ The law of excluded middle [LEM] amounts to $\vdash A \vee \neg A$, for every $A \in \mathscr{S}$.

${ }^{5}$ The methodology of a logical theory can be understood as analogous to Lakatos (1978) conception of methodologies for scientific research. However, the heuristic mechanisms developed for the logical theory are tied to the inferential and epistemic goals of the theory.

${ }^{6}$ See, e.g., Blake-Turner \& Russell (2018) for a similar account of the representational character of logical theories.

${ }^{7}$ See Laudan (1978).

${ }^{8}$ See Haack (1974).

${ }^{9}$ See da Costa et al. (1995) and Priest (2002).

${ }^{10}$ The principle of explosion (or of ex contradictione quodlibet) states that every formula follows from a contradiction, i.e. $\neg A, A \vdash B$.

${ }^{11}$ Note these views on contradictions might still be applied to other authors such as Routley (1980) and Batens (1999).

${ }^{12}$ In fact, Carnielli \& Rodrigues (2019) also motivate their reading of the data in terms of information, but still regard justification as the relevant item for the failure of PE.

${ }^{13}$ For an interpretation of the law of non-contradiction as a metaphysical principle, see Tahko (2009).

${ }^{14}$ One may also use the term 'should not' instead of 'cannot'.

${ }^{15}$ Note that even if different methodological constraints spread into different systems, they might still overlap. 
${ }^{16}$ Methodological underdetermination is understood here as a type of contrastive underdetermination relative to the methodology at play. Contrastive underdetermination states that for any body of evidence confirming a given hypothesis there might be other hypotheses explaining the same phenomenon. See Stanford (2017).

\section{Acknowledgments}

The author is grateful to many colleagues for their comments and suggestions about the ideas presented in this paper, particularly to Christian Straßer, Jonas Arenhart, Dunja Seselja, João Marcos, Heinrich Wansing and Hitoshi Omori. Two anonymous referees also provided many valuable suggestions during the review process. Gratitude also extends to the audience of the 4th Filomena Workshop, especially to the Bergen Logic Group. The present research was funded by a CAPES Scholarship. Process: 88887.161384/2017-00. 